\title{
MODELLI SPAZIO-TEMPORALI DI DIFFUSIONE, PREVISIONE E CONTROLLO DELLE EPIDEMIE DI COLERA: DAL SUDAFRICA AD HAITI
}

\author{
MARINO GATTO (*), LORENZO MARI (**), ANDREA RINALDO (***)
}

Nota presentata dal s.c. Marino Gatto

(Adunanza del 14 aprile 2011)

SuNTO. - Capire, predire e controllare le dinamiche del colera e di altre malattie idrotrasmesse sono obiettivi cruciali delle politiche sanitarie. Modelli quantitativi atti a prevedere l'evoluzione di un'epidemia e valutare i possibili scenari d'intervento sono particolarmente utili nel corso di un'emergenza sanitaria, quando è indispensabile agire tempestivamente e allocare risorse in modo efficiente. Tali modelli devono essere spazialmente espliciti, perché le epidemie di malattie idrotrasmesse mostrano pattern spaziali eterogenei. Sono qui presentati metodi e modelli epidemiologici spazialmente espliciti per la previsione in tempo reale di epidemie di colera e per la progettazione di strategie d'intervento per la loro prevenzione e controllo. Sono brevemente descritte le applicazioni a due epidemie di colera in Sudafrica e ad Haiti.

$* * *$

ABSTRACT. - Understanding, predicting and controlling the dynamics of cholera and other waterborne diseases are crucial goals of public health policies. Appropriate quantitative models to forecast epidemic development and to evaluate possible intervention

(") Dipartimento di Elettronica, Informazione e Bioingegneria, Politecnico di Milano, Italy. E-mail: marino.gatto@polimi.it

(**) Dipartimento di Elettronica, Informazione e Bioingegneria, Politecnico di Milano, Italy; Laboratory of Ecohydrology, École Polytechnique Fédérale de Lausanne, Switzerland.

(*t*a) Dipartimento di Ingegneria Civile, Edile ed Ambientale, Università di Padova, Italy; Laboratory of Ecohydrology, École Polytechnique Fédérale de Lausanne, Switzerland. 
scenarios would be specially needed during epidemic outbreaks, when preparedness and efficient allocation of resources are key to emergency management. Moreover, these tools ought to be spatially explicit, because waterborne disease epidemics usually display spatially inhomogeneous patterns. Here we describe spatially explicit epidemiological models and methods for real-time forecast of waterborne disease epidemics and design of intervention strategies for epidemic prevention and control. Applications to two cholera epidemics in South Africa and Haiti are briefly illustrated.

\section{INTRODUZIONE}

Le malattie idrotrasmesse sono infezioni che vengono prevalentemente contratte attraverso il contatto con o il consumo di acqua contaminata. Possono colpire uomini, animali e anche piante. Secondo la World Health Organization (WHO 2008) le malattie diarroiche sono al quinto posto tra le cause di morte $(3.7 \%)$ comportando più di 2 milioni di morti l'anno. La diarrea è al secondo posto tra le cause di morte dei bambini sotto i 5 anni (Fig. 1A). Quasi tutte le malattie diarroiche sono causate da microorganismi patogeni trasmessi attraverso l'acqua. Essi includono protozoi (ad es. Entamoeba bystolitica), batteri (ad es. Vibrio cholerae e Shigella dysenteriae) e virus (ad es. Rotavirus). Il colera è probabilmente la più conosciuta tra le malattie letali diarroiche, anche se altre malattie come il Rotavirus causano più decessi (circa 500.000 nel 2004 secondo WHO). L'andamento dei casi di colera nel ventennio passato (Fig. 1B) mostra un incremento in Africa e un decremento in America e Asia. L'epidemia recente più devastante è quella che ha colpito Haiti (Butler 2010; Enserink 2010, Barzilay 2013, MSPP 2013). Iniziata in ottobre 2010, ha avuto il picco in gennaio 2011, mostrando poi un andamento altalenante legato a forzanti idrologiche. All'inizio di maggio 2013 i casi complessivi sono stati più di 650.000 con oltre 8.000 morti.

Giova ricordare alcune delle caratteristiche del colera. La malattia è un'infezione intestinale acuta causata da un batterio, Vibrio cholerae, scoperto da Filippo Pacini nel 1854 durante un'epidemia verificatasi a Firenze. Il vibrione produce un'enterotossina, che può provocare diarrea profusa e morte per disidratazione. Oltre 155 sierotipi di vibrione sono conosciuti ma solamente i sierotipi O1 ed O139 (1992, Bangladesh) sono patogeni per l'uomo. Il batterio si trasmette per via oro-fecale, tramite l'ingestione di acqua o cibi contaminati. 
Generalmente la dose infettante del batterio è piuttosto elevata (circa un milione di vibrioni). I principali serbatoi sono il comparto acquatico, specie l'acqua salata o salmastra, di cui è un componente naturale, spesso in associazione con fito e zooplancton, e il comparto umano. Il periodo di incubazione della malattia varia tra 2 ore e 5 giorni. Un'importante caratteristica è che il 70-80\% degli infetti sono asintomatici, ma producono batteri nelle escrezioni fecali per 7-14 giorni. Tra la restante percentuale, costituita dai sintomatici, solo il 20\% sviluppa la malattia in forma acuta con diarrea profusa e disidratazione. La mortalità è bassa in rapporto all'enorme numero di casi che si possono verificare, generalmente intorno all' $1 \%$. Il tempo di guarigione è di circa 5 giorni. Gli infetti, sia sintomatici sia asintomatici, sviluppano un'immunità non permanente la cui durata non è facilmente determinabile, ma si aggira intorno a qualche anno.

Capire, predire e controllare le dinamiche del colera e di altre malattie idrotrasmesse sono obiettivi cruciali delle politiche sanitarie. Modelli quantitativi atti a prevedere l'evoluzione di un'epidemia e valutare i possibili scenari d'intervento sono particolarmente utili nel corso di un'emergenza sanitaria, quando è indispensabile agire tempestivamente e allocare risorse in modo efficiente. Tali modelli devono essere spazialmente espliciti, perché le epidemie di malattie idrotrasmesse mostrano configurazioni spaziali eterogenee. Tuttavia, modelli di questo tipo non sono comuni nella letteratura sul colera, né sono abitualmente impiegati nella definizione delle strategie di intervento.
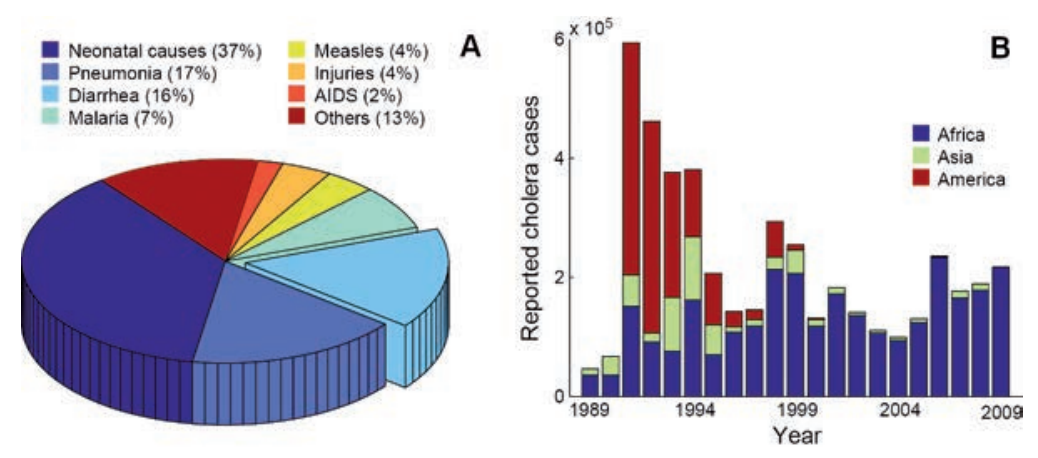

Fig. 1 - A) Cause più comuni di morte tra i bambini sotto is anni (dalla Fig. 2 in UNICEF/WHO 2009); B) casi di colera dichiarati alla WHO nel ventennio passato. 


\section{I MODELLI MATEMATICI DEL COLERA}

Il modello fondativo fu proposto sul finire degli anni 1970 (Capasso e Paveri-Fontana 1979) e sviluppato per l'epidemia di colera nel Mediterraneo del 1973. Codeço (2001) introdusse poi un modello che considerava tre equazioni differenziali ordinarie dove, in aggiunta ai comparti di suscettibili (S) e infetti (I) che caratterizzano i modelli microparassitologici tradizionali (Anderson e May 1991), una equazione descriveva la dinamica dei batteri (B) in acqua. Estensioni sono state proposte per tener conto ad es. dell'iperinfettività dei batteri appena escreti (Hartley et al. 2006), o l'interazione preda-predatore tra i batteri e i loro fagi (Jensen et al. 2006). Forzanti stagionali e climatiche sono state introdotte (Colwell 1996; Pascual et al. 2002; Lipp et al. 2002; Koelle et al. 2005; Righetto et al. 2012) per includere l'influenza di diversi regimi idrologici sul decorso temporale delle esplosioni epidemiche o di endemismi.

Tutti questi modelli si basano su approssimazioni di campo medio, cioè non considerano la componente spaziale della diffusione della malattia. Tale componente, come mostrato da molti dati nel mondo, è importante perché le epidemie mostrano andamenti spazialmente disomogenei. $\grave{E}$ pertanto fondamentale considerare in maniera esplicita altri meccanismi, come la propagazione spaziale specificamente legata alla mobilità dell'uomo (o di altri animali) o alle vie d'acqua. Aggiungere la componente spaziale è una delle maggiori sfide della modellistica ecologica (p.e. Walsh et al. 2005; Real et al. 2005; Tilman e Kareiva 1997; Diekmann e Heesterbeeck 2000). I primi modelli spaziali erano un'estensione degli schemi classici a spazio continuo con trasmissione della malattia su scala locale tramite un nucleo appropriato, o operatori Laplaciani che la diffondevano tra i vicini (Kendall 1965; Durrett e Levin 1994; Sato et al. 1994; Rand et al. 1995).

La componente spaziale dei processi epidemiologici può essere utilmente descritta anche tramite reti spaziali i cui nodi siano comunità locali e gli archi connessioni spaziali tra essi. Pur essendo estremamente generale nella sua formulazione, tale idea trova un'applicazione specifica nelle reti fluviali, che possono vedersi come corridoi ecologici per la biodiversità, le popolazioni e le malattie idrotrasmesse (RodriguezIturbe et al. 2009). I corridoi fluviali possono infatti propagare patogeni dalle regioni costiere a quelle interne e viceversa, e dai siti epidemici 
d'entroterra alle aree confinanti (Bertuzzo et al. 2008; Akanda et al. 2009; Bertuzzo et al. 2010). Le reti fluviali conformano la distribuzione delle comunità locali lungo i fiumi e impongono vincoli anisotropici di connettività spaziale che risultano essenziali per una corretta caratterizzazione dei processi ecologici negli ecosistemi fluviali (Bertuzzo et al. 2007). L'analisi di tali caratteristiche è quindi particolarmente importante per lo studio delle epidemie di colera. Inoltre, come detto sopra, in molti casi gli individui infetti sono asintomatici e dunque possono inavvertitamente diffondere la malattia muovendosi tra una comunità non ancora infetta e una già infettata.

\section{Modelli Su RETE Di MALATtie IDROTRASMESSE}

Recentemente, è stato proposto un approccio spazialmente esplicito per modellizzare la propagazione di patogeni tramite le acque di superficie come un processo reattivo di trasporto lungo reti introducendo dunque il meccanismo primario di infezione (Bertuzzo et al. 2008, 2010). La mobilità umana è descritta sia attraverso modelli basati sulla diffusione (Righetto et al. 2011) che di tipo gravitazionale (Bertuzzo et al. 2011; Tuite et al. 2011; Chao et al. 2011; Mari et al. 2011; Reiner et al. 2012).

Una struttura generale (Fig. 2) per descrivere le dinamiche delle malattie idrotrasmesse nello spazio e nel tempo è stata sviluppata congiuntamente da ricercatori dell'École Polytechnique Fédérale de Lausanne, del Politecnico di Milano e della Princeton University. Questo approccio è basato su reti multi-strato (Mari et al. 2011), in cui i nodi corrispondono alle comunità umane composte da persone suscettibili, infette e guarite con accesso a una riserva d'acqua potenzialmente contaminata (Codeço 2001), mentre gli archi rappresentano strati di connettività spaziale fornita dal trasporto dei patogeni lungo reti fluviali (Bertuzzo et al. 2010) e dalla mobilità umana (Mari et al. 2012a).

Grazie alla sua flessibilità e applicabilità a casi reali, questo approccio si è mostrato molto efficace nella descrizione di epidemie di colera passate, in particolare quella del KwaZulu-Natal in Sudafrica (Mari et al. 2012b, Fig. 3) e in corso (Rinaldo et al. 2012, Fig. 4), così come nella definizione di condizioni di insorgenza per epidemie di malattie idrotrasmesse (Gatto et al. 2012). 


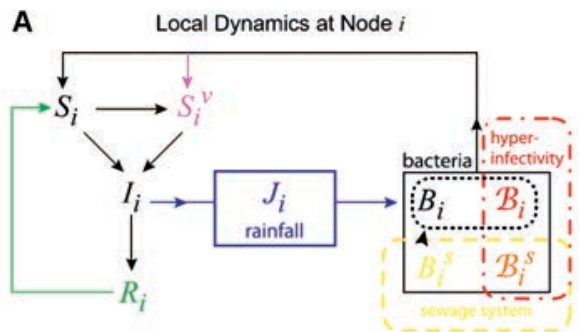

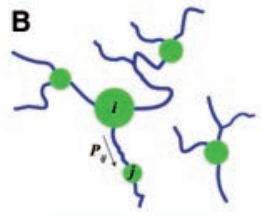

Hydrologic Network
C

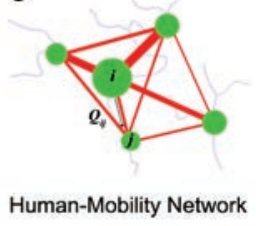

Fig. 2 - Elementi del modello per le malattie idrotrasmesse.

A) Diagramma a blocchi delle dinamiche epidemiologiche locali; B) rappresentazione schematica della rete idrolologica e $C$ ) di mobilità umana. I nodi (cerchi verdi) rappresentano comunità umane di diversa grandezza. Le connessioni tra i nodi sono dovute a vie d'acqua, attraverso le quali i patogeni si diffondono per trasporto attivo e passivo, o al movimento di individui suscettibili o infetti.

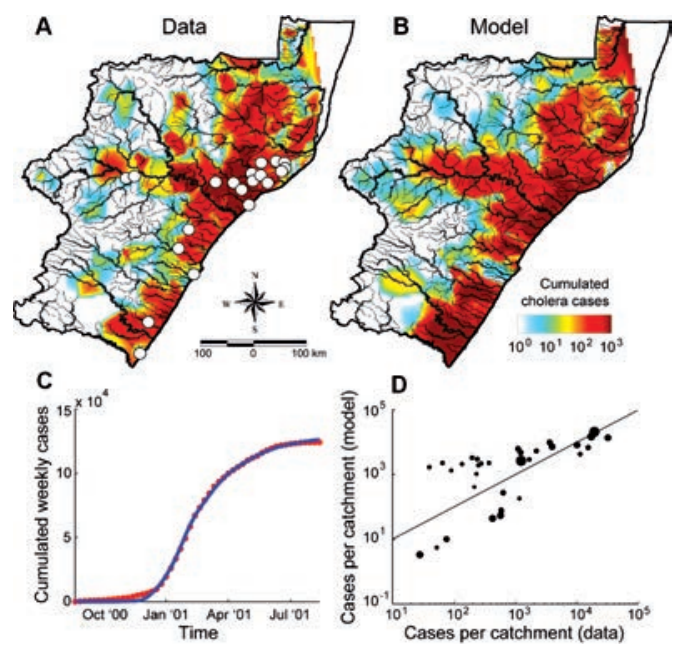

Fig. 3 - Evoluzione di un'epidemia di colera in KwaZulu-Natal simulata col modello descritto in Mari et al. (2012b).

A) Casi riportati e B) simulati nel periodo 2000-2001; $i$ punti bianchi nel pannello $A$ individuano la distribuzione spaziale dei primi casi riportati; $C$ ) evoluzione temporale dell'epidemia (rosso: casi reali; blu: casi simulati); D) distribuzione spaziale dei casi. 


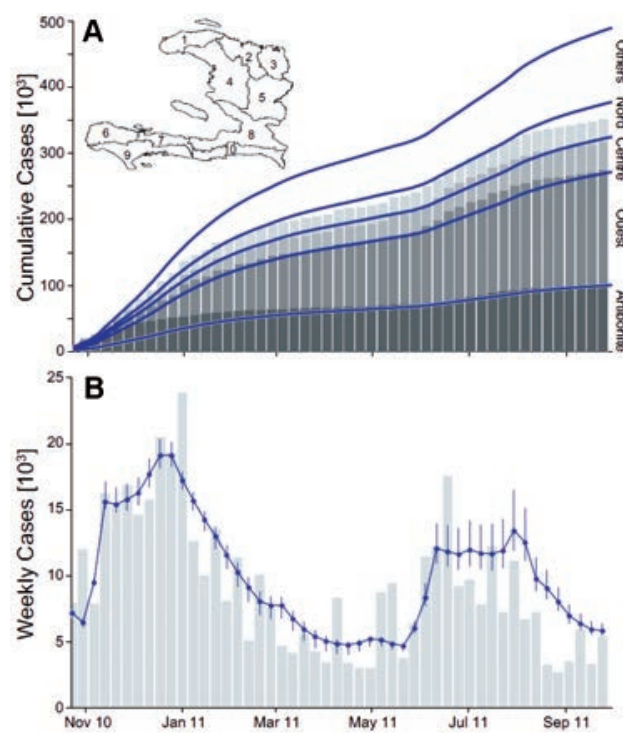

Fig. 4 - Evoluzione dell'epidemia di colera ad Haiti simulata tra ottobre 2010 e settembre 2011 col modello descritto in Rinaldo et al. (2012).

A) Casi cumulati (grigio: casi reali; blu: casi simulati) nei quattro dipartimenti maggiormente colpiti dal colera (Artibonite, Ouest, Centre e Nord, numerati come 4, 8, 5 e 2 nella mappa); B) casi settimanali totali.

\section{CONCLUSiOni}

La simultanea inclusione della rete idrologica e della rete di mobilità umana ha permesso di descrivere e predire la distribuzione spaziale e temporale delle epidemie in Sudafrica e ad Haiti. Analogamente le reti spaziali sono state utilizzate anche per caratterizzare configurazioni di biodiversità (Muneepeerakul et al. 2008) e diffusione di specie invasive (Mari et al. 2011) dando prova d'essere uno strumento flessibile per l'analisi dei sistemi ecologici.

I modelli spazialmente espliciti possono essere decisivi in termini operativi, in particolare per la previsione dell'evoluzione di un'epidemia e per la progettazione di opportune strategie di intervento. Le misure che possono essere adottate per il controllo delle malattie umane idrotrasmesse possono essere strategiche (prevenzione e sorveglianza) o tattiche (controllo dell'epidemia; Wisner e Adams 2002). Compromessi (costi 
contro efficacia) e vincoli (risorse economiche, disponibilità di personale o materiale sanitario) sono sempre presenti nella realtà. I modelli spaziali verranno quindi utilizzati in futuro per identificare in modo quantitativo le strategie di intervento più efficienti nello spazio e nel tempo, sia ex ante che in tempo reale. L'ottimalità delle decisioni potrà essere valutata attraverso metodi multicriteriali (Belton e Stewart 2003) basati su opportune funzioni obiettivo (che tengano conto di vite salvate, ospedalizzazioni evitate, costi di intervento) i cui valori possano essere valutati dal modello. Uno schema di gestione adattativa (Allan e Stankey 2009), che permette l'assimilazione in tempo reale di nuove informazioni disponibili, potrà essere utilizzato per ridurre l'incertezza delle previsioni del modello e delle strategie per il controllo dell'epidemia.

\section{RIFERIMENTI BIBLIOGRAFICI}

A. Akanda, S. Jutla, S. Islam, Dual peak cholera transmission in Bengal Delta: a bydroclimatological explanation, Geophysical Research Letters 36 (2009), L19401.

C. Allan, G.H. Stankey, Adaptive environmental management: a practitioner's guide, Dordrecht, Springer, 2009.

R.M. Anderson, R.M. May, Infectious diseases of bumans: dynamics and control, Oxford, Oxford University Press, 1991.

E.J. Barzilay, N. Schaad, R. Magloire, K.S. Mung, J. Boncy, G.A. Dahourou, E.D. Mintz, M.W. Steenland, J.F. Vertefeuille, J.W. Tappero, Cholera surveillance during the Haiti epidemic - The first 2 years, The New England Journal of Medicine 368 (2013), 599-609.

V. Belton, T. Stewart, Multiple criteria decision analysis: an integrated approach, Dordrecht, Kluwer Academic Publishers, 2002.

E. Bertuzzo, A. Maritan, M. Gatto, I. Rodriguez-Iturbe, A. Rinaldo, River networks and ecological corridors: reactive transport on fractals, migration fronts, bydrochory, Water Resources Research 43 (2007), W04419.

E. Bertuzzo, M. Gatto, A. Maritan, S. Azaele, I. Rodriguez-Iturbe, A. Rinaldo, On the space-time evolution of a cholera epidemic, Water Resources Research 44 (2008), W01424.

E. Bertuzzo, R. Casagrandi, M. Gatto, I. Rodriguez-Iturbe, A. Rinaldo, On spatially explicit models of cholera epidemics, Journal of the Royal Society Interface 7 (2010), 321-333.

E. Bertuzzo, L. Mari, L. Righetto, M. Gatto, R. Casagrandi, M. Blokesch, I. RodriguezIturbe, A. Rinaldo, Prediction of the spatial evolution and effects of control measures for the unfolding Haiti cholera outbreak, Geophysical Research Letters 38 (2011), L06403.

D. Butler, Cholera tightens grip on Haiti, Nature 468 (2010), 483-484. 
V. Capasso, S.L. Paveri-Fontana, A mathematical model for the 1973 cholera epidemic in the European Mediterranean region, Revue d'Epidemiologie et de la Santé Publique 27 (1979), 121-132.

D. Chao, M. Halloran, I. Longini, Vaccination strategies for epidemic cholera in Haiti with implications for the developing world, Proceedings of the National Academy of Sciences of the United States of America 108 (2011), 7081-7085.

C.T. Codeço, Endemic and epidemic dynamics of cholera: the role of the aquatic reservoir, BMC Infectious Diseases 1 (2001), 1.

R.R. Colwell, Global climate and infectious disease: the cholera paradigm, Science 274 (1996), 2025-2031.

O. Diekmann, J. Heesterbeek, Mathematical epidemiology of infectious diseases: model building, analysis and interpretation, Chichester, Wiley, 2000.

R. Durrett, S.A. Levin, The importance of being discrete (and spatial), Theoretical Population Biology 46 (1994), 363-394.

M. Enserink, Infectious diseases: Haiti's outbreak is latest in cholera's new global assault, Science 330 (2010), 738-739.

M. Gatto, L. Mari, E. Bertuzzo, R. Casagrandi, L. Righetto, I. Rodriguez-Iturbe, A. Rinaldo, Generalized reproduction numbers and the prediction of patterns in waterborne disease, Proceedings of the National Academy of Sciences of the United States of America 109 (2012), 19703-19708.

D. Hartley, J. Morris, D. Smith, Hyperinfectivity: a critical element in the ability of $\mathrm{V}$. cholerae to cause epidemics?, PLoS Medicine 3 (2006), 63-69.

M. Jensen, S. Faruque, J. Mekalanos, B. Levin, Modeling the role of bacteriophage in the control of cholera outbreaks, Proceedings of the National Academy of Sciences of the United States of America 103 (2006), 4652-4657.

D. Kendall, Mathematical models of the spread of infection, In: Mathematics and computer science in biology and medicine, Medical Research Council, London, H. M. Stationery Office, 1965.

K. Koelle, X. Rodó, M. Pascual, Md. Yunus, G. Mostafa, Refractory periods and climate forcing in cholera dynamics, Nature 436 (2005), 696-700.

E. Lipp, A. Huq, R.R. Colwell, Effects of global climate on infectious disease: the cholera model, Clinical Microbiology Reviews 15 (2002), 757-770.

L. Mari, E. Bertuzzo, R. Casagrandi, M. Gatto, S.A. Levin, I. Rodriguez-Iturbe, A. Rinaldo, Hydrologic controls and anthropogenic drivers of the zebra mussel invasion of the Mississippi-Missouri river system, Water Resources Research 47 (2011), W03523.

L. Mari, E. Bertuzzo, L. Righetto, R. Casagrandi, M. Gatto, I. Rodriguez-Iturbe, A. Rinaldo, On the role of buman mobility in the spread of cholera epidemics: towards an epidemiological movement ecology, Ecohydrology 5 (2012a), 531-540.

L. Mari, E. Bertuzzo, L. Righetto, R. Casagrandi, M. Gatto, I. Rodriguez-Iturbe, A. Rinaldo, Modelling cholera epidemics: the role of waterways, buman mobility and sanitation, Journal of the Royal Society Interface 9 (2012b), 376-388. 
Ministère de la Santé Publique et de la Population, Republique d'Haiti, Rapports journaliers du MSPP sur l'évolution du choléra en Haiti, http://www.mspp.gouv.ht/ site/index.php (01/05/2013).

R. Muneepeerakul, E. Bertuzzo, H. Lynch, W. Fagan, A. Rinaldo, I. Rodriguez-Iturbe, Neutral metacommunity models predict fish diversity patterns in MississippiMissouri basin, Nature 453 (2008), 220-224.

M. Pascual, M.J. Bouma, A.P. Dobson, Cholera and climate: revisiting the quantitative evidence, Microbes and Infection 4 (2002), 237-245.

D.A. Rand, M. Keeling, H.B. Wilson, Invasion, stability and evolution to criticality in spatially extended, artificial host-pathogen ecologies, Proceedings of the Royal Society of London B 259 (1995), 55-63.

L.A. Real, J.C. Henderson, R. Biek, J. Snaman, T. Lambert Jack, J.E. Childs, E. Stahl, L. Waller, R. Tinline, S. Nadin-Davis, Unifying the spatial population dynamics and molecular evolution of epidemic rabies virus, Proceedings of the National Academy of Sciences of the United States of America 102 (2005), 12107-12111.

R.C. Reiner, A.A. King, M. Emch, M. Yunus, A.S.G. Faruque, M. Pascual, Highly localized sensitivity to climate forcing drives endemic cholera in a megacity, Proceedings of the National Academy of Sciences of the United States of America 109 (2012), 2033-2036.

L. Righetto, E. Bertuzzo, R. Casagrandi, M. Gatto, I. Rodriguez-Iturbe, A. Rinaldo, Modelling buman movement in cholera spreading along fluvial systems, Ecohydrology 4 (2011), 49-55.

L. Righetto, R. Casagrandi, E. Bertuzzo, L. Mari, M. Gatto, I. Rodriguez-Iturbe, A. Rinaldo, The role of aquatic reservoir fluctuations in long-term cholera patterns, Epidemics 4 (2012), 33-42.

A. Rinaldo, E. Bertuzzo, L. Mari, L. Righetto, M. Blokesch, M. Gatto, R. Casagrandi, M. Murray, S. Vesenbeckh, I. Rodriguez-Iturbe, Reassessment of the 2010-2011 Haiti cholera outbreak and multi-season projections via inclusion of rainfall and waning immunity, Proceedings of the National Academy of Sciences of the United States of America 109 (2012), 6602-6607.

I. Rodriguez-Iturbe, R. Muneepeerakul, E. Bertuzzo, S. Levin, A. Rinaldo, River networks as ecological corridors: a complex systems perspective for integrating bydrologic, geomorphologic and ecologic dynamics, Water Resources Research 45 (2009), W01413.

K. Sato, H. Matsuda, A. Sasaki, Pathogen invasion and host extinction in lattice structured populations, Journal of Mathematical Biology 32 (1994), 251-268.

D. Tilman, P. Kareiva, Spatial ecology: the role of space in population dynamics and interspecific interactions, Princeton, Princeton University Press, 1997.

A. Tuite, J. Tien, M. Eisenberg, D. Earns, J. Ma, D. Fisman, Cholera epidemic in Haiti, 2010: using a transmission model to explain spatial spread of disease and identify optimal control interventions, Annals of Internal Medicine 154 (2011), 593-601.

United Nations Children's Fund, World Health Organization, The state of the world's children, New York, UNICEF, 2009. 
P.D. Walsh, R. Biek, L.A. Real, Wave-like spread of Ebola Zaire, PloS Biology 3 (2005), 1-8.

B. Wisner, J. Adams, Environmental bealth in emergencies and disasters: a practical guide, Genève, WHO Press, 2002.

World Health Organization, The global burden of disease: 2004 update, Genève, WHO Press, 2008. 